\title{
Modeling of the near-field distribution of pollutants coming from a coastal outfall
}

\author{
T. P. Lyubimova ${ }^{1}$, B. Roux ${ }^{2}$, S. Luo ${ }^{2}$, Y. N. Parshakova ${ }^{1}$, and N. S. Shumilova ${ }^{1}$ \\ ${ }^{1}$ Institute of Continuous Media Mechanics Ural Branch of RAS, Perm, Russia \\ ${ }^{2}$ M2P2, UMR 7340, CNRS Aix-Marseille University and Ecole Centrale, Marseille, France
}

Correspondence to: T. P. Lyubimova (lubimova@psu.ru)

Received: 7 December 2012 - Revised: 25 February 2013 - Accepted: 27 March 2013 - Published: 26 April 2013

\begin{abstract}
The present study concerns the 3-D distribution of pollutants emitted from a coastal outfall in the presence of strong sea currents. The problem is solved using the nonlinear Reynolds-averaged Navier-Stokes equations in the framework of the $k-\varepsilon$ model. The constants of the logarithmic law for the vertical velocity profile in the bottom boundary layer are obtained by processing experimental data from acoustic Doppler current profilers (ADCPs). The near-field distribution of pollutants at different distances from the diffuser is obtained in terms of the ambient flow velocity (steady or with tidal effect) and outfall discharge characteristics. It is shown that even in the case where the effluent density is substantially lower than the ambient sea water density the plume can impact the seabed, creating a risk of pollution of removable bottom sediments.
\end{abstract}

\section{Introduction}

The oceans and seas are the ultimate sink for most pollution, largely because of their huge volume and assimilating capacities. No place in the world's seas is immune from pollution, as ocean currents transport pollutants to the far corners of the world. Coastal waters act as a natural buffer zone due to their intermediate position between the deep sea and human activities on land. In recent years, interest in environmental issues, in particular, in the preservation of water quality in coastal regions, has steadily increased. Here we consider the nearfield dilution of industrial effluent discharged by a coastal outfall. We attempt to evaluate the environmental risk associated with the discharge of effluent into seabed sediments in the case of a buoyant effluent, which is less dense than the receiving marine water. In reality, bottom sediments can ac- cumulate pollutants in a matter of months and then migrate unexpectedly in response to, for example, storm-driven currents. Under the action of alternating semi-diurnal tidal currents, these movable bottom sediments can be accumulated beneath the diffuser pipe, which causes an obstruction of the interval between the pipe and the sea bottom. The objective of this work is to investigate the distribution of pollutants in the bottom boundary layer (BBL), which is treated to be a layer adjacent to the seabed. In this layer, the flow is affected by the processes occurring at the boundary. Strong gradients of physical, chemical, and biological properties may occur in this layer as well Bowden (1978). The thickness of the BBL is generally of the order of meters above the seabed. There has been a large amount of work devoted to the study of the BBL and sediment dynamics on the shoreface, e.g., Holmedal et al. (2003), Kim et al. (2000) and Styles et al. (2000). We study the BBL dynamics in the near-field of a multiport ocean outfall with submerged buoyant jets discharging in the vicinity of the sea bottom into the ambient flows. Special attention is given to the problem of interaction between the effluent plume and the sea bottom. We intend to determine the conditions for bottom attachment (Coanda attachment) by analyzing the ambient flow and discharge characteristics.

\section{Problem formulation}

We consider a circular ocean multiport outfall with vertical discharge placed near the seabed perpendicular to the main direction of the ambient current, as shown in Fig. 1. The diffuser involves two hundred discharge jets (with circular opening of diameter $d=0.04 \mathrm{~m}$ ) spaced every $5 \mathrm{~m}$ along the pipe. 


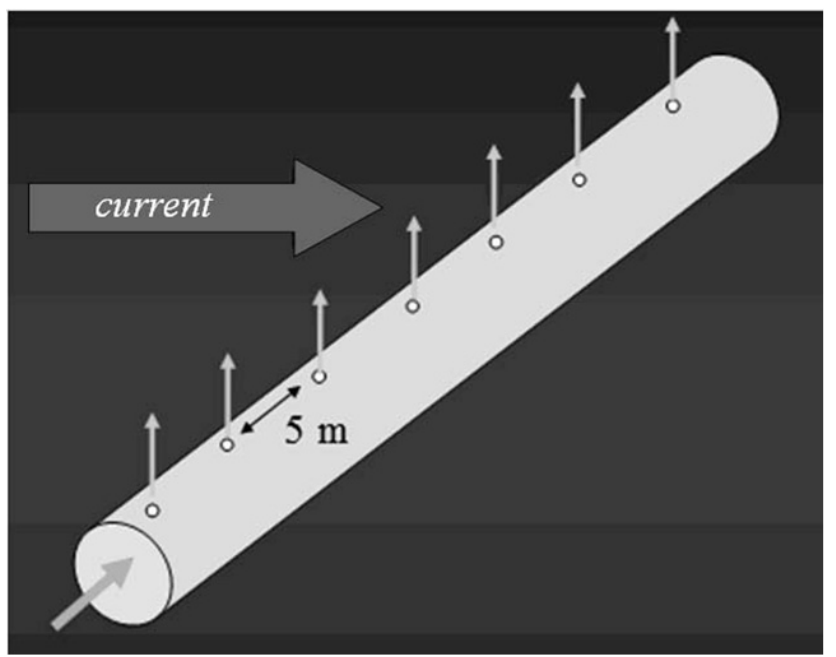

Fig. 1. Schematic description of the multiport ocean outfall (the current direction being parallel to the mean shoreline and perpendicular to the diffuser; the discharge jets from circular ports are vertical)

Two main configurations are considered, when the interval under the diffuser is empty or filled by removable bottom sediments. The first case corresponds to the nominal configuration, the second one would simulate the risk of sediment accumulation under the diffuser due to alternate semi-diurnal tidal currents (the pipe behaving as a barrier). For both cases we carry out the numerical simulation of the pollution of coastal waters from a point source which corresponds to one port (circular opening with a diameter $d$ ) of the ocean outfall diffuser. The diffuser pipe has a circular section of diameter $d$ at the height $L$ from the seabed. We consider a buoyant effluent which is discharged vertically at average velocity. The velocity of the ambient current is denoted $\boldsymbol{V}$. Diffuser characteristics are tube of constant diameter: $70 \mathrm{~cm}$ (external); $60 \mathrm{~cm}$ (internal); unidirectional multiport system (200 holes of diameter $40 \mathrm{~mm}$, spaced at intervals of $5 \mathrm{~m}$ ); vertical discharge; at a height $y=1.20 \mathrm{~m}$ from the seabed. The discharge velocity of the effluent, which depends on the mass flow rate and decreases along the diffuser; the mean values are $0.50 \mathrm{~m} \mathrm{~s}^{-1}, 1.35 \mathrm{~m} \mathrm{~s}^{-1}, 3.00 \mathrm{~m} \mathrm{~s}^{-1}$.

\section{Mathematical model}

To get a realistic near-field distribution of pollutant a nonlinear 3-D unsteady approach is needed. We implement Reynolds-averaged Navier-Stokes equations:

$\frac{\partial \rho}{\partial t}+\frac{\partial}{\partial x_{i}}\left(\rho u_{i}\right)=0$

$\frac{\partial}{\partial t}\left(\rho u_{i}\right)+\frac{\partial}{\partial x_{j}}\left(\rho u_{i} u_{j}\right)=\frac{\partial}{\partial x_{j}}\left[\mu\left(\frac{\partial u_{i}}{\partial x_{j}}+\frac{\partial u_{j}}{\partial x_{i}}-\frac{2}{3} \delta_{i j} \frac{\partial u_{l}}{\partial x_{l}}\right)\right]+$ $+\frac{\partial}{\partial x_{j}}\left[\mu_{t}\left(\frac{\partial u_{i}}{\partial x_{j}}+\frac{\partial u_{j}}{\partial x_{i}}\right)-\frac{2}{3}\left(\rho k+\mu_{t} \frac{\partial u_{l}}{\partial x_{l}}\right) \delta_{i j}\right]+\rho g_{i}-\frac{\partial p}{\partial x_{i}}$.
Here, $\rho$ is the density, $x_{i}$ are coordinates (we use Cartesian coordinate system), $u_{i}$ are the velocity components, $\mu$ is the kinematic viscosity, $\mu_{t}$ is the turbulent viscosity. The turbulence kinetic energy $\mathrm{k}$ and rate of its dissipation $\varepsilon$ are obtained from the following transport equations:

$$
\begin{aligned}
& \frac{\partial}{\partial t}(\rho k)+\frac{\partial}{\partial x_{i}}\left(\rho k u_{i}\right)=\frac{\partial}{\partial x_{j}}\left[\left(\mu+\frac{\mu_{t}}{\sigma_{k}}\right) \frac{\partial k}{\partial x_{j}}\right]+G_{k}+G_{b}-\rho \varepsilon \\
& \frac{\partial}{\partial t}(\rho \varepsilon)+\frac{\partial}{\partial x_{i}}\left(\rho \varepsilon u_{i}\right)=\frac{\partial}{\partial x_{j}}\left[\left(\mu+\frac{\mu_{t}}{\sigma_{\varepsilon}}\right) \frac{\partial \varepsilon}{\partial x_{j}}\right]+ \\
& +C_{1 \varepsilon} \frac{\varepsilon}{k}\left(G_{k}+C_{3 \varepsilon} G_{b}\right)-C_{2 \varepsilon} \rho \frac{\varepsilon^{2}}{k} .
\end{aligned}
$$

In these equations, $G_{k}$ represents the generation of turbulence kinetic energy due to the mean velocity gradients $G_{k}=$ $\mu_{t} S^{2}$, where $S$ is the modulus of the mean strain rate tensor, defined as $S=\sqrt{2 S_{i j} S_{i j}}, S_{i j}=\frac{1}{2}\left(\frac{\partial u_{j}}{\partial x_{i}}+\frac{\partial u_{i}}{\partial x_{j}}\right), G_{b}$ is the turbulence kinetic energy due to buoyancy, which is calculated as

$G_{b}=g_{i}\left(\beta \frac{\mu_{t}}{\operatorname{Pr}_{t}} \frac{\partial T}{\partial x_{i}}+\frac{1}{\rho}\left(\frac{\partial \rho}{\partial c}\right) \mu_{s t} \frac{\partial c}{\partial x_{i}}\right)$,

i.e. taking into account the dependence of the density on temperature and concentration, $\beta$ is the thermal expansion coefficient, $\mu_{s t}$ is the parameter of density stratification due to the concentration, $\operatorname{Pr}_{t}$ is the turbulent Prandtl number, $g_{i}$ is the component of the gravitational vector in $i$-th direction; $G_{1 \varepsilon}, C_{2 \varepsilon}$, and $C_{3 \varepsilon}$ are constants, $\sigma_{k}$ and $\sigma_{\varepsilon}$ are the turbulent Prandtl numbers for $k$ and $\varepsilon$, respectively. The turbulent viscosity $\mu_{t}$ is computed as $\mu_{t}=\rho C_{\mu} k^{2} / \varepsilon$, where $C_{\mu}$ is a constant. The model constants $\operatorname{Pr}_{t}, G_{1 \varepsilon}, C_{2 \varepsilon}, C_{\mu}, \sigma_{k}$ and $\sigma_{\varepsilon}$ were taken to have the following values: $\operatorname{Pr}_{t}=0.85, C_{1 \varepsilon}=1.44$, $C_{2 \varepsilon}=1.92, C_{\mu}=0.09, \sigma_{k}=1.0, \sigma_{\varepsilon}=1.3$. Turbulent heat transport is modeled using the concept of the Reynolds analogy to turbulent momentum transfer. The "modeled" energy equation is thus written as

$$
\frac{\partial}{\partial t}(\rho E)+\frac{\partial}{\partial x_{i}}\left[u_{i}(\rho E+p)\right]=\frac{\partial}{\partial x_{j}}\left(k_{\mathrm{eff}} \frac{\partial T}{\partial x_{j}}+u_{i}\left(\tau_{i j}\right)_{\mathrm{eff}}\right),
$$

where $E=c h+\frac{p}{\rho}$ is the total energy, $h=C_{p} T$ is sensible enthalphy, $k_{\text {eff }}$ is the effective thermal conductivity and $\left(\tau_{i j}\right)_{\text {eff }}$ is the deviatoric stress tensor defined by

$\left(\tau_{i j}\right)_{\mathrm{eff}}=\mu_{\mathrm{eff}}\left(\frac{\partial u_{j}}{\partial x_{i}}+\frac{\partial u_{i}}{\partial x_{j}}\right)-\frac{2}{3} \mu_{\mathrm{eff}} \frac{\partial u_{k}}{\partial x_{k}} \delta_{i j}$,

where $\mu_{\mathrm{eff}}=k_{\mathrm{eff}} \operatorname{Pr} / C_{p}$ is the effective viscosity, effective thermal conductivity is given by $\kappa_{\text {eff }}=\kappa+C_{p} \mu_{t} / \operatorname{Pr}_{t}$, where $\kappa$ is the thermal conductivity. The equation of mass transfer is

$$
\frac{\partial}{\partial t}(\rho c)+\frac{\partial}{\partial x_{i}}\left(\rho u_{i} c\right)=-\frac{\partial}{\partial x_{i}} J_{i},
$$

where $\boldsymbol{J}=-\left(\rho D+\mu_{t} / \mathrm{Sc}_{t}\right) \nabla c$, is the mass flux, $S c_{t}=$ $\mu_{t} / \rho D_{t}$ is the turbulent Schmidt number, and the turbulent 


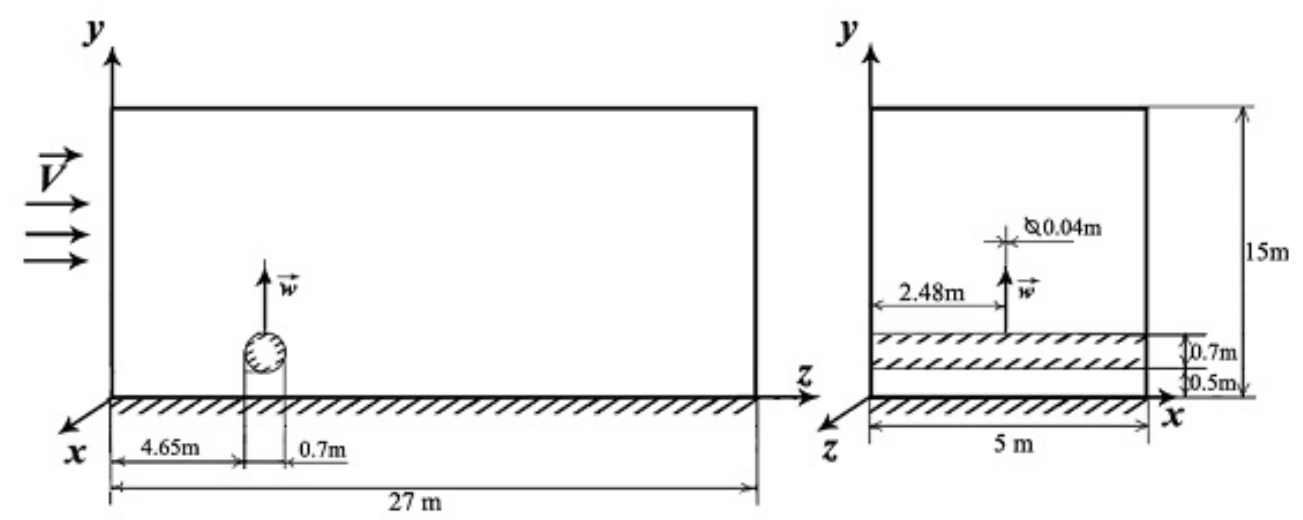

Fig. 2. Schematic representation of the problem and coordinates: longitudinal view (along the ambient current); transversal view (along the diffuser).

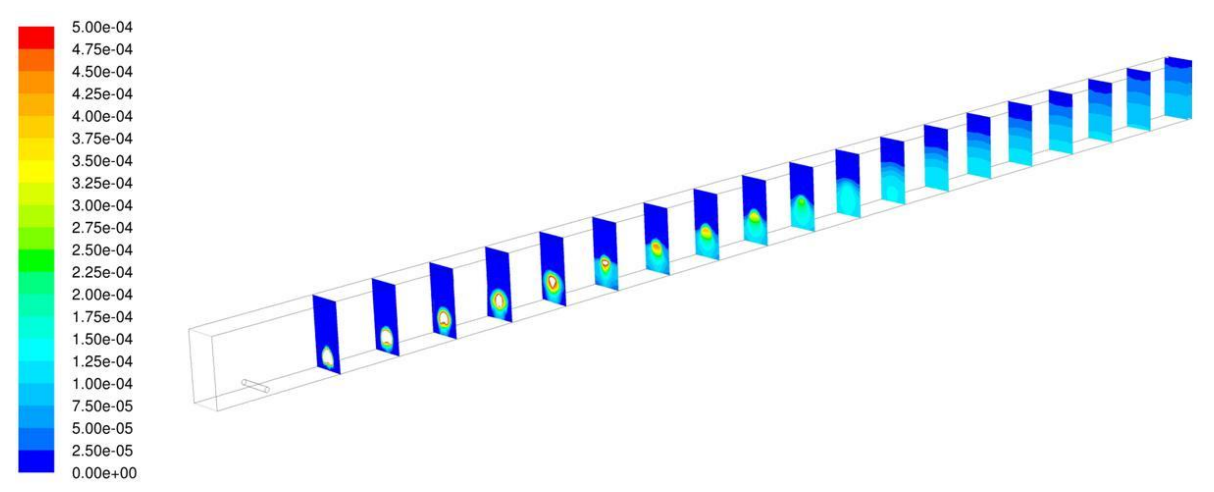

Fig. 3. Concentration fields in vertical cross sections perpendicular to the ambient current at different distances from the diffuser: 20,30 , 40 , $\ldots, 200 \mathrm{~m}$. Ambient current velocity $V=0.55 \mathrm{~m} \mathrm{~s}^{-1}$. The unit of concentration is wt $\%$.

diffusion parameter $D_{t}$ describes turbulent mass transfer. The dependence of density on temperature and concentration is defined according to the linear law

$\rho=\rho_{0}-\rho_{0} \beta\left(T-T_{0}\right)-\rho_{0} \beta_{c}\left(c-c_{0}\right)$,

where $\rho_{0}, T_{0}, c_{0}$ are the density, temperature and concentration of the sea water.

\subsection{Boundary conditions}

We set the no-slip condition, zero mass flux condition and fixed temperature on the rigid boundaries (seabed and pipe surface):

$u_{x}=u_{y}=u_{z}=0, \quad \frac{\partial c}{\partial n}=0, \quad T=T_{0}$.

At the pipe exit (port) we impose the constant ejection velocity (vertical), pollutant concentration and temperature of the discharged liquid:

$u_{x}=u_{z}=0, \quad u_{y}=w, \quad T=T_{e}, \quad c=c_{e}$.
At the entrance of computational domain we impose the velocity of ambient current, in which the $z$-component (perpendicular to the outfall axis) is the only non-zero component depending on the vertical coordinate $\boldsymbol{V}=\{0, V(y), 0\}$; the concentration and temperature are set equal to the background temperature and background concentration of pollutant in the sea water.

$z=0: \quad u_{x}=u_{y}=0, \quad u_{z}=V, \quad T=T_{0}, \quad c=c_{0}$

The conditions on the other boundaries of the computational domain are

$\frac{\partial u_{i}}{\partial n}=0 \quad(i=x, y, z), \quad \frac{\partial T}{\partial n}=0, \quad \frac{\partial c}{\partial n}=0$.

\subsection{Accounting for the bottom boundary layer (BBL)}

Since a multiport diffuser is placed near the seabed (i.e., in the bottom boundary layer), it is important to analyze the effect of the ambient BBL dynamics. For that purpose, we consider two types of the vertical profile for a steady ambient current $V(y)$ : (a) constant in $y$ (i.e., without BBL effect), and (b) a logarithmic profile of the ambient velocity: $V(y)=a+b \ln (y)$, where the constants $a$ and $b$ are 


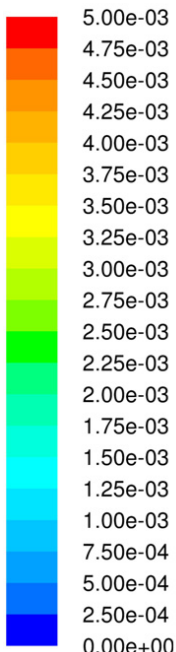

(a)
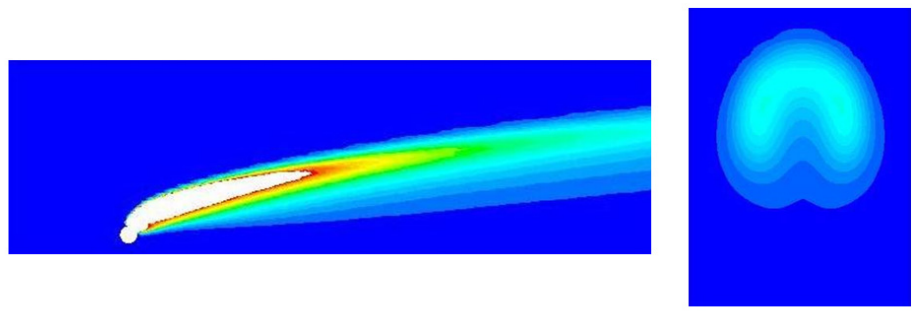

(b)
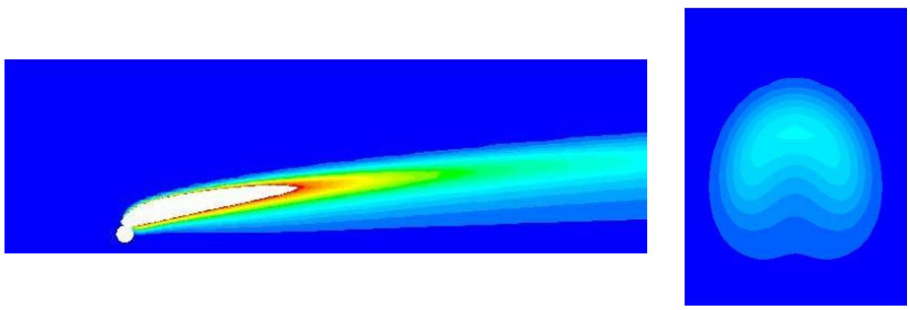

Fig. 4. Projections of 3-D pollutant concentration field: (a) constant velocity of the ambient flow : $V=0.15 \mathrm{~m} \mathrm{~s}^{-1}$ (b) logarithmic BBL profile $V(y)=0.144+0.033 \ln (\mathrm{y}) \mathrm{m} \mathrm{s}^{-1}$. Longitudinal view: vertical cross section passing through the port center $(\mathrm{x}=2.5 \mathrm{~m})$, and transversal view at a $\mathrm{z}$-distance of $22 \mathrm{~m}$ from the port. The unit of concentration is $\mathrm{wt} \%$.

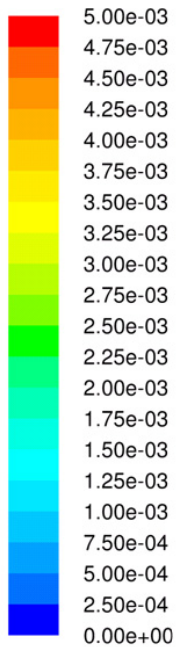

(a)
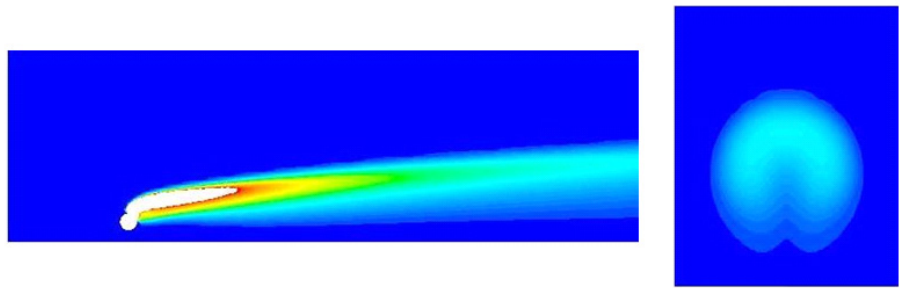

(b)
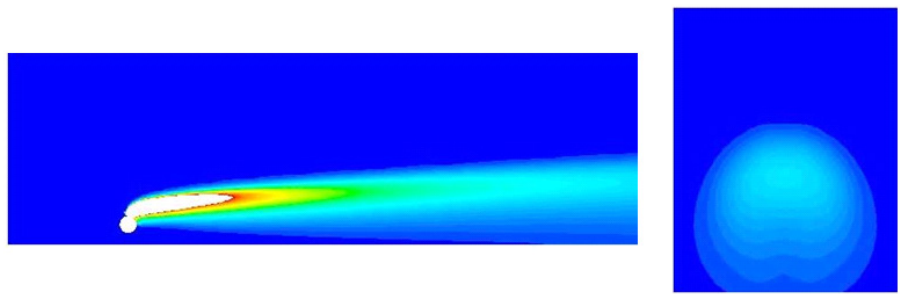

Fig. 5. Projections of 3-D pollutant concentration field: (a) constant velocity of the ambient flow : $V=0.25 \mathrm{~m} \mathrm{~s}^{-1}$ (b) logarithmic BBL profile $V(y)=0.241+0.049 \ln (\mathrm{y}) \mathrm{m}$ s. Longitudinal view: vertical cross section passing through the port center $(\mathrm{x}=2.5 \mathrm{~m})$ and transversal view at a $\mathrm{z}$-distance of $22 \mathrm{~m}$ from the port. The unit of concentration is wt $\%$.

extracted from experimental data obtained from Acoustic Doppler Current Profiler (ADCP), in the water column at the place of the outfall. When ADCP profile is available, it permits to save computation time by reducing the computation domain upwards. To analyze the role of BBL formulation, we compare the results of numerical simulation for a constant ambient velocity with the results obtained for a logarithmic profile normalized according to the following rule: at a distance of $1.2 \mathrm{~m}$ from the seabed (which corresponds to the height at which the discharge exit is located) the values of the velocity in both cases coincide (i.e., $\left.V_{2}(1.2)=a \ln (1.2)+b=V_{1}\right)$. The values of the examined velocity $V_{1}=$ const, $\mathrm{m} \mathrm{s}^{-1}$ are $0.05,0.15,0.25,0.35$ and corresponding values of $V_{2}(y)=a+b \ln (y), \mathrm{m} \mathrm{s}^{-1}$ are $0.048+$ $0.014 \ln (y), 0.144+0.033 \ln (y), 0.241+0.049 \ln (y)$, and $0.340+0.062 \ln (y)$. For numerical calculation we use the following parameters of the real system: environmental characteristics (ambient sea water): density $\rho_{0} \approx$ $1024 \mathrm{~kg} \mathrm{~m}^{-3}$, temperature $T_{0}=297 \mathrm{~K}$, current speed (perpendicular to the diffuser) $V=0.1 \div 0.6 \mathrm{~m} \mathrm{~s}^{-1}$, concentration $c_{0}=0 \mathrm{wt} \%$; effluent characteristics: density at the diffuser exit: $\rho_{e} \approx 1004 \mathrm{~kg} \mathrm{~m}^{-3}$, effluent temperature $T_{e}=313 \mathrm{~K}$, mean discharge speed, $w=1.35 \mathrm{~m} \mathrm{~s}^{-1}$, and concentration $c_{0}=1 \mathrm{wt}, \%$; physical parameters: kinematical viscosity 


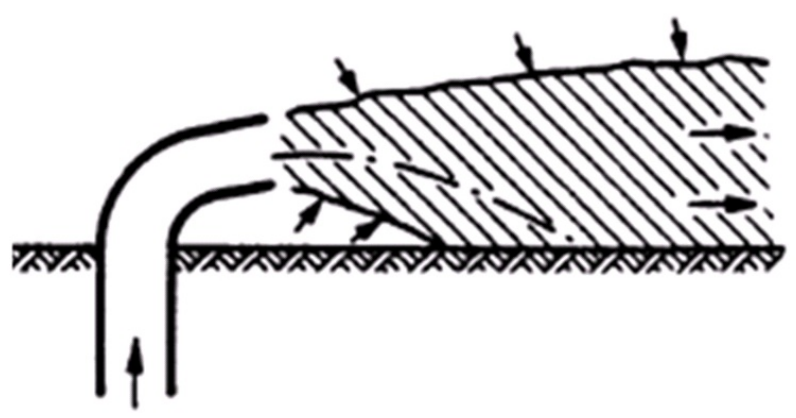

Fig. 6. Example of Coanda attachment conditions for submerged jets discharging near boundaries (after (Jirka et al., 1996)).

$\mu=9.33529 \cdot 10^{-7} \mathrm{~m}^{2} \mathrm{~s}^{-1}$, thermal expansion coefficient $\beta=2.5 \cdot 10^{4} 1 / K$, thermal conductivity $k=0.6 \mathrm{~W} /(m \cdot \mathrm{K})$, heat capacity $C_{p}=3995 \mathrm{~J} /(\mathrm{kg} \cdot \mathrm{K})$, mass diffusivity $D=$ $10^{-9} \mathrm{~m}^{2} \mathrm{~s}^{-1}$, turbulent Schmidt number $S c_{t}=0.7$, and Prandtl number $\operatorname{Pr}=1$. Numerical simulations were carried out using the commercial software package ANSYS Fluent. We checked the effect of the computational domain and mesh sizes by considering three configurations: dimension $5 \mathrm{~m} \times 27 \mathrm{~m} \times 10 \mathrm{~m}$, mesh $31 \times 86 \times 45$; dimension $5 \times 600 \times 10 \mathrm{~m}$, and mesh $31 \times 416 \times 48$; dimension $5 \times 850 \times 10 \mathrm{~m}$, mesh $31 \times 430 \times 48$. On the basis of these test calculations, for implementation of the basic calculations we used the computational domain $5 \mathrm{~m} \times 900 \mathrm{~m} \times 10 \mathrm{~m}$ and the mesh size $31 \times 430 \times 48$. We implemented the mesh refinement with minimal mesh size $0.004 \mathrm{~m}$ near the hole; the maximal mesh size is $4 \mathrm{~m}$.

\section{Numerical results}

\subsection{Nominal configuration (surelevated pipe)}

Let us consider the case where the velocity of the ambient current is steady.

\subsubsection{The steady case (with and without BBL effect)}

We first examined the nominal configuration (surelevated pipe; Fig. 2).

The calculations for this configuration were performed for ambient current speed varied from $V=0.1=\mathrm{m} \mathrm{s}^{-1}$ to $V=0.6 \mathrm{~m} \mathrm{~s}^{-1}$. An example of plume development in $\mathrm{z}$ direction is presented in Fig. 3; transversal cross sections of the pollutant concentration are presented for constant inlet velocity $V=0.55 \mathrm{~m} \mathrm{~s}^{-1}$, dimensions of computational domain are $5 \mathrm{~m} \times 205 \mathrm{~m} \times 10 \mathrm{~m}$ and mesh dimensions are $31 \times 230 \times 48$. The BBL effect is illustrated in Figs. 4 and 5 , where one can see the patterns of pollutant propagation in the vertical cross sections that are parallel and perpendicular to the ambient flow for two types of the vertical profile of ambient velocity: (a) constant in y, (b) logarithmic in $y$, and two values of the ambient current velocity: $V=0.15 \mathrm{~m} \mathrm{~s}^{-1}$ and $V=0.25 \mathrm{~m} \mathrm{~s}^{-1}$. It is seen that the obtained results recover the classical horseshoe structure of a turbulent round jet deviated by a crossflow (see, for ex. Muppidi and Mahesh (2007); Fig. 3). The jet deflects in the direction of the crossflow. Additionally, a pair of counter-rotating vortices is generated.

In Figs. 8 and 9, we present the pollutant concentration fields in vertical cross sections parallel and perpendicular to the ambient flow obtained numerically for the case with blockage at $V=0.15 \mathrm{~m} \mathrm{~s}^{-1}$ and $V=0.25 \mathrm{~m} \mathrm{~s}^{-1}$ with and without taking into account the BBL formulation.

For a higher speed of ambient current Fig. 5, there is an evidence of bottom attachment very similar to the one described by Jirka et al. (1996) and named Coanda attachment, for submerged buoyant jets discharging in the vicinity of the water bottom into a stagnant or flowing ambient. Two types of dynamic interaction processes have been described by Jirka et al. (1996) that lead to rapid attachment of the effluent plume to the water bottom. These are wake attachment forced by the receiving water's crossflow or Coanda attachment forced by the entrainment demand of the effluent jet itself. The latter, which is due to low pressure effects as the jet periphery is close to the water bottom, is schematically shown in Fig. 6. For the logarithmic velocity profile, the effect of bottom attachment is more pronounced than in the case of constant velocity; the bottom attachment by the pollutant spot occurs at smaller distance from the discharge point.

\subsubsection{Configuration with deposit under the pipe (block- age effect)}

The configuration examined above is idealized. In reality, the bottom sediments can be accumulated under the pipe due to the alternate semi-diurnal tidal current, as the pipe constitutes a barrier to the bottom sediment transport. To analyze this case we introduced a new configuration with a rigid wall under the pipe (Fig. 7).

Let us first discuss the results obtained with blockage at the constant ambient velocity profile. The simulations show that in this case a vortex is created in the pollutant wake behind the pipe, which leads to a shift of plume towards the bottom (the plume attaches the bottom just behind the pipe) (Figs. 8a-9a). The existence of pollutant wake behind the pipe is caused by the reverse flow arising in the wake of solid obstacle. This reverse flow promotes accumulation of the pollutant under the pipe that is why, if the pollutant accumulation mechanism is switched on, it will be self-amplified. In the case of the logarithmic velocity profile, accumulation of pollutants near the bottom also takes place; the pollutant spot at the bottom occupies the area larger than in the case of the constant ambient velocity profile. The propagation of pollutants downstream of the ambient flow and the maximal values of pollutant concentration are observed at smaller 


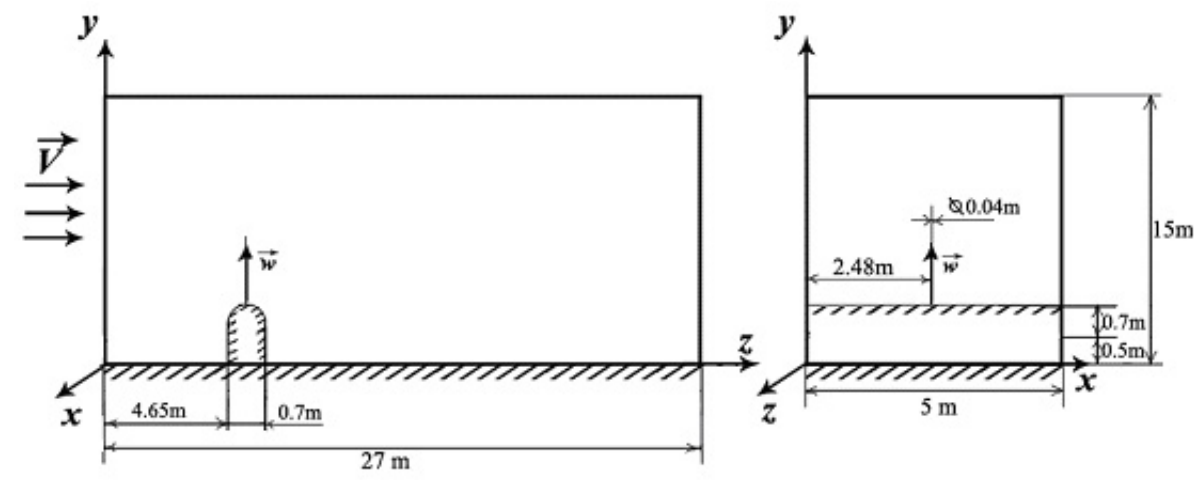

Fig. 7. Configuration with the deposit under the pipe: longitudinal view (along the ambient current); transversal view (along the diffuser).

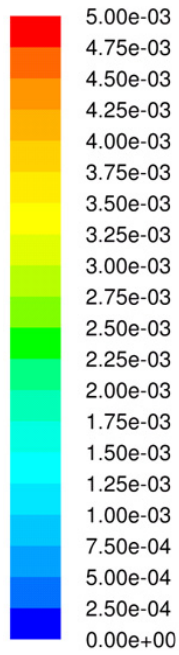

(a)
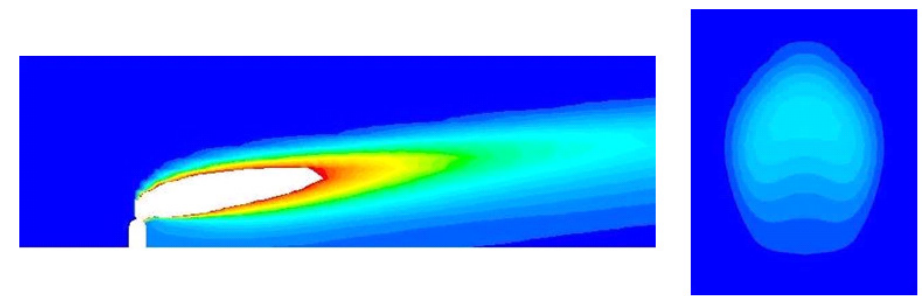

(b)
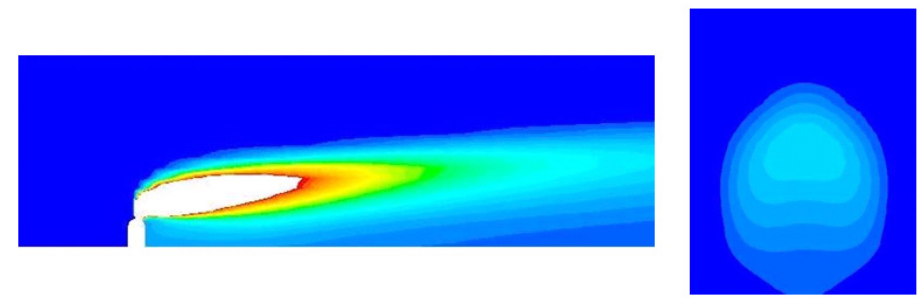

Fig. 8. Projections of 3-D pollutant concentration field: (a) constant velocity of the ambient flow: $V=0.15 \mathrm{~m} \mathrm{~s}^{-1}$ (b) logarithmic BBL profile $V(y)=0.144+0.033 \ln (\mathrm{y}) \mathrm{m} \mathrm{s}$. Longitudinal view: vertical cross section passing through the port center $(\mathrm{x}=2.5 \mathrm{~m})$ and transversal view at a $\mathrm{z}$-distance of $22 \mathrm{~m}$ from the port. The unit of concentration is wt $\%$.

distances from the bottom. This is attributed to the fact that, in the case of the logarithmic ambient velocity profile, the vortex created just behind the blockage has higher intensity and occupies the greater area. As in the case of the constant velocity profile, this vortex shifts the pollutant plume downward.

\subsection{The unsteady case (tidal effect)}

Consider now a situation where the ambient current is timedependent: the coastal current is parallel to the shoreline and is driven alternatively by a semi-diurnal tide $(T=12 \mathrm{~h}$ $25 \mathrm{~min}=44700 \mathrm{~s}$ ). This ambient current is still assumed to be perpendicular to the outfall; it is also assumed to be periodical in time as follows: $V(y, t)=-V(y) \sin (2 \pi t / T)$, where $V(y)$ corresponds to the BBL formulation mentioned above. Both configurations, with and without blockage, have been analyzed. The longitudinal pollutant distribution has been computed for several cycles. The results presented be- low are given for four instants of a well-established cycle (Figs. 10,11). We chosen instants for which the inlet velocity of the sea current is successively equal to $0.15,0.25$, 0.35 , and $0.45 \mathrm{~m} \mathrm{~s}^{-1}$. Each set of figures contains a vertical cross section, and a few top views at different distances above seabed. All the calculations are realized with the BBL formulation.

In the case without blockage, the top views for the distances from the bottom $0 \mathrm{~m}$ and $0.5 \mathrm{~m}$ are not shown, since they would be completely blue with the concentration being lower than $2.5 \cdot 10^{-4} \mathrm{wt} \%$. In both situations (without and with blockage), the numerical simulations show that, for a semi-diurnal periodic ambient current, the plume will attach the sea bed at some time intervals during which the current is of the order of $0.25 \mathrm{~m} \mathrm{~s}^{-1}$ as in the steady case. The plume impact is more important in the case with blockage. 


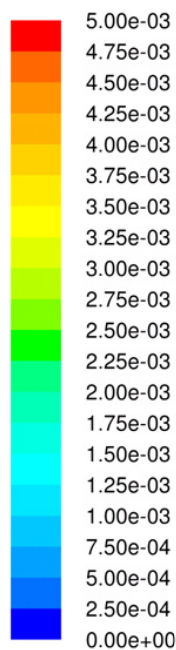

(a)
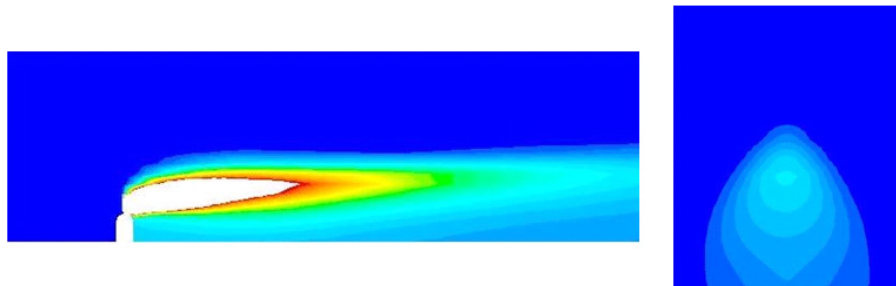

(b)
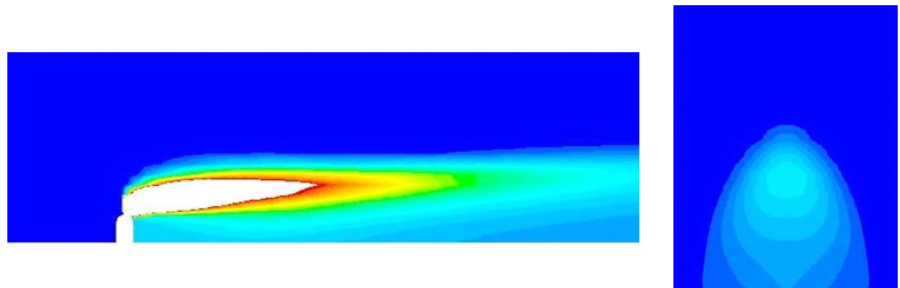

Fig. 9. Projections of 3-D pollutant concentration field: (a) constant velocity of the ambient flow: $V=0.25 \mathrm{~m} \mathrm{~s}^{-1}$ (b) logarithmic BBL profile $V(y)=0.241+0.049 \ln (\mathrm{y}) \mathrm{m} \mathrm{s}^{-1}$. Longitudinal view: vertical cross section passing through the port center $(\mathrm{x}=2.5 \mathrm{~m})$ and transversal view at a $\mathrm{z}$-distance of $22 \mathrm{~m}$ from the port. The unit of concentration is wt $\%$.

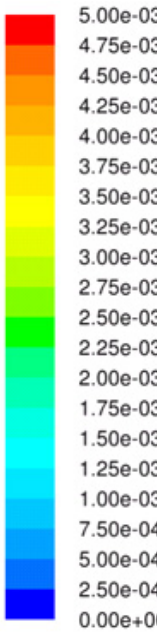

(a)
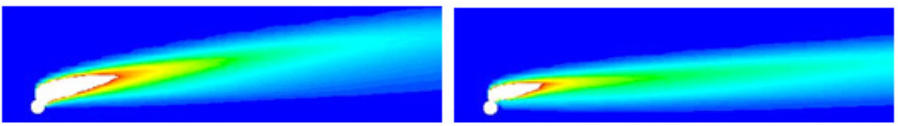

(b)
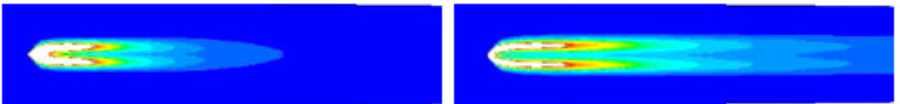

(c)
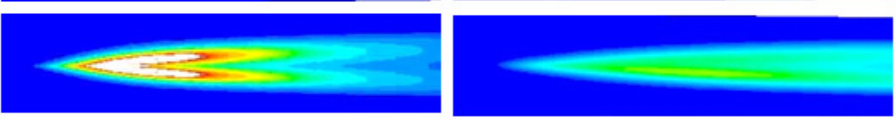

(d)
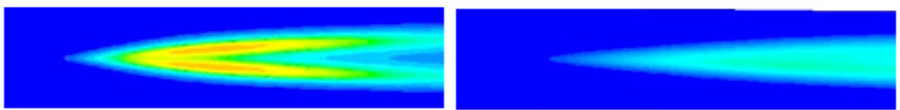

(A) $\mathrm{V} \approx 0.15 \mathrm{~m} / \mathrm{s} ; \mathrm{t}=3800 \mathrm{sec}$

(B) $\mathrm{V} \approx 0.25 \mathrm{~m} / \mathrm{s} ; \mathrm{t}=5500 \mathrm{sec}$

(a)
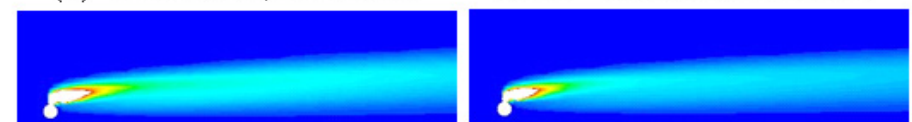

(b)
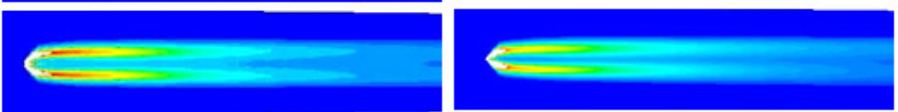

(c)
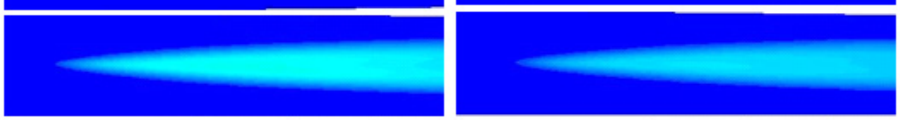

(d)

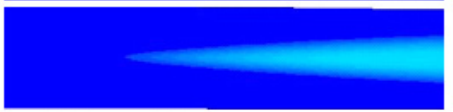

(C) $\mathrm{V} \approx 0.35 \mathrm{~m} / \mathrm{s} \mathrm{t}=3800 \mathrm{sec}$

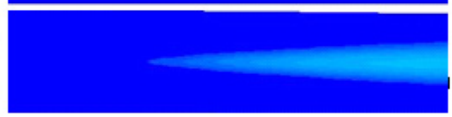

(D) $\mathrm{V} \approx 0.45 \mathrm{~m} / \mathrm{s} ; \mathrm{t}=5500 \mathrm{sec}$

Fig. 10. Longitudinal views of pollutant concentration at four instants, for a periodic ambient current, BBL formulation without blockage; (a) vertical cross section passing through the port center, (b-d) top view at different distances above the seabed: (b) $1.2 \mathrm{~m}$; (c) $2.5 \mathrm{~m}$; and (d) $3 \mathrm{~m}$. 


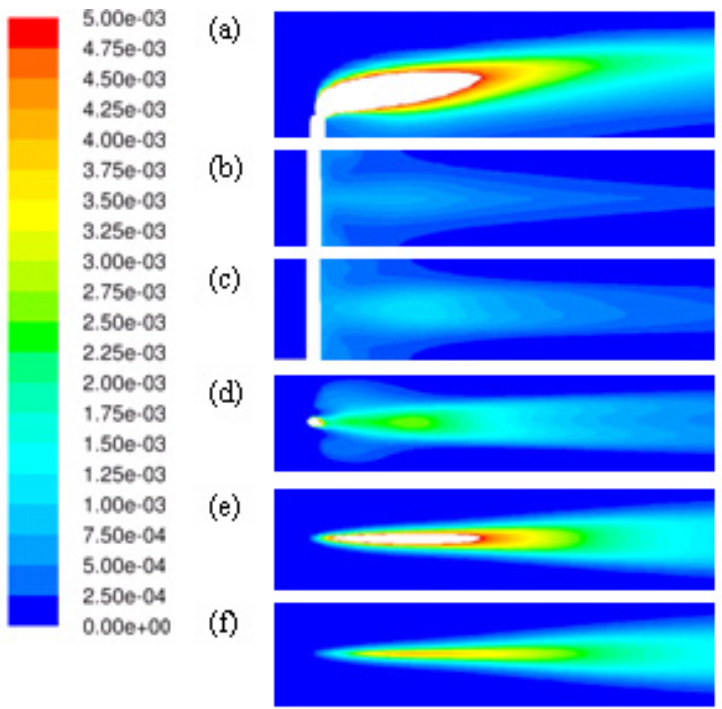

(A) $\mathrm{V} \approx 0.15 \mathrm{~m} / \mathrm{s} \mathrm{t}=3800 \mathrm{sec}$

(a)

(b)

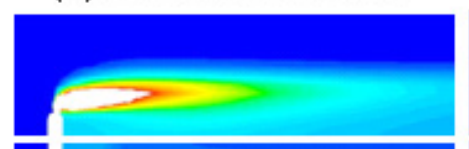

(c)

(d)

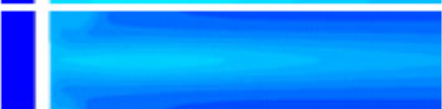

(e)

(f)
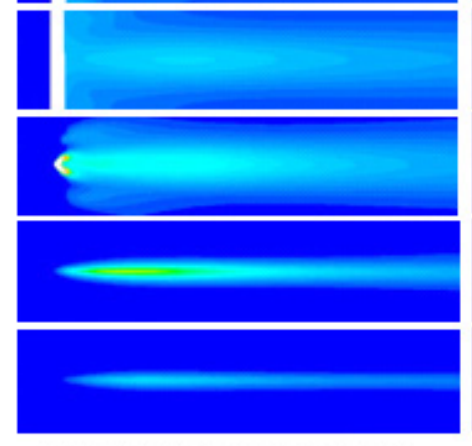

(C) $\mathrm{V} \approx 0.35 \mathrm{~m} / \mathrm{s} ; \mathrm{t}=7500 \mathrm{sec}$
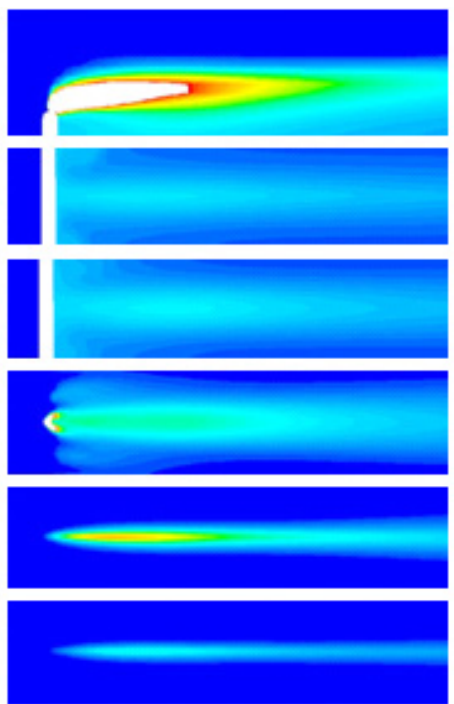

(B) $\mathrm{V} \approx 0.25 \mathrm{~m} / \mathrm{s} ; \mathrm{t}=5500 \mathrm{sec}$

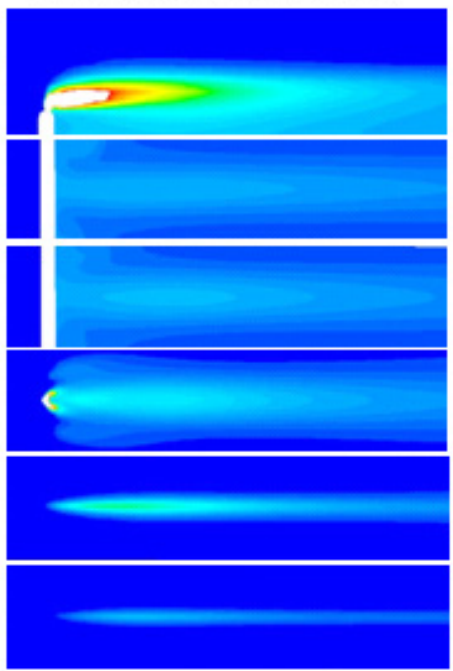

(D) $\mathrm{V} \approx 0.45 \mathrm{~m} / \mathrm{s} ; \mathrm{t}=11000 \mathrm{sec}$

Fig. 11. Longitudinal views of pollutant concentration at four instants, for a periodic ambient current, BBL formulation, with blockage; (a) vertical cross section passing through the port center, (b-f) top view at different distances above the seabed: (b) $0 \mathrm{~m}$; (c) $0.5 \mathrm{~m}$; (d) $1.2 \mathrm{~m}$; (e) $2.5 \mathrm{~m}$; (f) $3 \mathrm{~m}$.

\subsection{General discussion about bottom attachment mech- anisms: effect of $L$ and $w$}

Here, according to the kind suggestion of one referee, a generalization of results of simulations is attempted to determine range of parameters when effluent is attached to bottom. In addition to the effect of the ambient flow velocity, $V$, we consider the effects the height of the pipe, $L$, and the effect of discharge velocity, $w$, on the longitudinal evolution of the pollutant concentration at the seabed. These effects are considered in the case of a steady ambient flow, with different velocities $V$ ranging from $0.25 \mathrm{~m} \mathrm{~s}^{-1}$ to $0.55 \mathrm{~m} \mathrm{~s}^{-1}$. The effect of the pipe height, is shown in Fig. 12, for a discharge velocity $w=1.35 \mathrm{~m} \mathrm{~s}^{-1}$. It can be seen that a substantial pollutant concentration is found at a shorter distance of the pipe when $L$ is decreased from $L=0.5 \mathrm{~m}$ to $L=0.25 \mathrm{~m}$ (Fig. 12b). That means that in the case of when the height of the pipe is too small, a maximum of the pollutant concentration at the seabed has to be expected at a very short $y$-distance of the pipe. This is coherent with the situation observed in the of blockage (Fig. 12c), which is worse. In addition, we observe that the pollutant concentration at the bottom is found to be higher for the lower velocity of the ambient flow. The effect of the discharge velocity, $w$, is shown in Fig. 13, for $L=0.5 \mathrm{~m}$. As could be expected, pollutant concentration at the seabed grows with the increase of $w$ (steady case). 

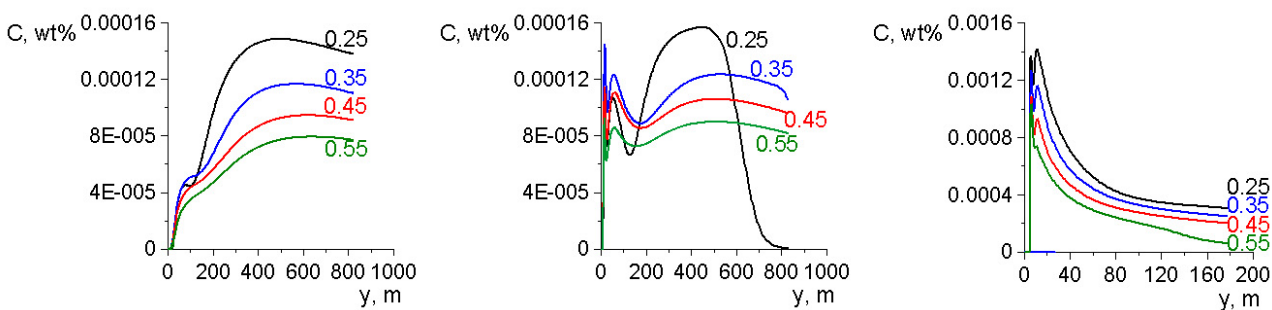

Fig. 12. Longitudinal evolution of the pollutant concentration at the seabed; (left) $L=0.5 \mathrm{~m}$; (middle) $L=0.25$ and (right) in the case of blockage.
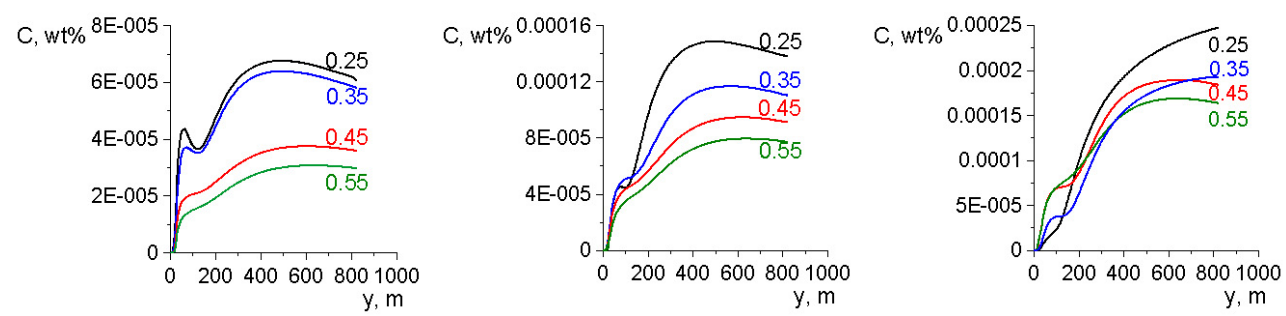

Fig. 13. Longitudinal evolution of the pollutant concentration at the seabed for different discharge velocities; (left) $w=0.5 \mathrm{~m} \mathrm{~s}{ }^{-1} ;($ middle $)$ $w=1.35 \mathrm{~m} \mathrm{~s}^{-1}$ and (right) $w=3 \mathrm{~m} \mathrm{~s}^{-1}$.

\section{Conclusions}

Determination of the risk of benthic pollution in the nearfield of the diffuser is an important environmental issue. A high risk of such pollution exists because the plume impacts the seabed, where bottom sediments (sand, silt, etc.) can accumulate pollutants in a matter of days or months. Moreover, the contaminated sediments can be moved away by water from the site into other areas under extreme events, e.g., storms. The main results of this paper can be summarized as follows: for the buoyant effluent discharged into the marine environment at a depth greater than $15 \mathrm{~m}$, we can expect a bottom attachment of the plume, even when the effluent density is substantially lower than the receiving sea water $\left(1004 \mathrm{~kg} \mathrm{~m}^{3}\right.$ vs. $1024 \mathrm{~kg} \mathrm{~m}^{3}$ ). This attachment (Coanda) effect has been exhibited with a simple model in which the ambient current velocity is constant at the upward limit of the computational domain (steady approach). In the case where ADCP measurements are available, we may use BBL formulation for the ambient current; in this case the bottom attachment is observed as well. In the case of blockage under the diffuser pipe, this effect of bottom attachment has been found to be much more pronounced. When the height of the pipe (without blockage) is reduced (from $0.5 \mathrm{~m}$ to $0.25 \mathrm{~m}$ ) a maximum of the bottom concentration occurs at a very short $y$-distance of the pipe. This is coherent with our results in the case of blockage. The longitudinal evolution of the pollutant concentration at the seabed has been evaluated for different discharge velocities; the bottom concentration increases with $w$. We have performed calculations using the time-dependent approach that takes into account the tidal effect (sinusoidal approximation). The obtained results confirm the main features obtained in the case of a steady state model, and in particular the fact that the Coanda attachment effect is more pronounced in the case of blockage under the diffuser pipe. In the case of blockage, or in the case of when the height of the pipe is too small, a maximum of the pollutant concentration at the seabed has to be expected at a very short $y$-distance of the pipe. This means that, in the near-field of the diffuser, the benthic materials (sand, silt, etc.) will accumulate pollutants in a matter of days or months, and then under different extreme events (storms) these contaminated sediments could move to more vulnerable sites. Calculations have indicated that such an impact becomes possible when the ambient current reaches about $0.25 \mathrm{~m} \mathrm{~s}^{-1}$.

Acknowledgements. This study was partly supported by the Province Sud de Nouvelle Caledonie for the French side and by Ural Branch of Russian Academy of Sciences (Program 12-1-1026) for the Russian side. The Authors also wish to thank reviewers for their pertinent remarks.

Edited by: E. Pelinovsky

Reviewed by: K. Klevannyy and one anonymous referee

\section{References}

Bowden, K. F.: Physical problems of the benthic boundary layer, Geophys. Surveys, 3, 255-296, 1978.

Holmedal, L. E., Myrhaug, D., and Rue, H.: The sea bed boundary layer under random waves plus current, Continental Shelf Res., 23, 717-750, 2003. 
Jirka, G. H., Doneker, R. L., and Hinton, S. W.: User's manual for CORMIX: a hydrodynamic mixing zone model and decision support system for pollutant discharges into surface waters, DeFrees Hydraulics Laboratory, Cornell University: Ithaca, NY, 1996.

Kim, S.-C., Friedrichs, C. T., Maa, J. P.-Y., and Wright, L. D.: Estimating bottom stress in tidal boundary layer from acoustic Doppler velocimeter data, J. Hydraulic Eng., 399-406, 2000.
Muppidi, S. and Mahesh, K.: Direct numerical simulation of round turbulent jets in crossflow, Fluid Mech., 574, 59-84, 2007.

Styles, R. and Glenn, S. M.: Modelling stratified wave and current bottom boundary layer on the continental shelf, J. Geophys. Res., 105, 24119-24139, 2000. 